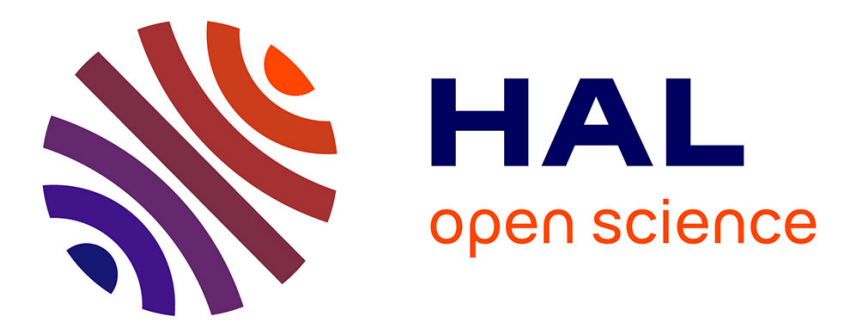

\title{
Q-plates micro-arrays for parallel processing of the photon orbital angular momentum
}

Charles Loussert, Kateryna Kushnir, Etienne Brasselet

\section{To cite this version:}

Charles Loussert, Kateryna Kushnir, Etienne Brasselet. Q-plates micro-arrays for parallel processing of the photon orbital angular momentum. Applied Physics Letters, 2014, 105 (12), pp.121108 (1-4). 10.1063/1.4895706 . hal-01068128

\section{HAL Id: hal-01068128 \\ https://hal.science/hal-01068128}

Submitted on 23 Feb 2016

HAL is a multi-disciplinary open access archive for the deposit and dissemination of scientific research documents, whether they are published or not. The documents may come from teaching and research institutions in France or abroad, or from public or private research centers.
L'archive ouverte pluridisciplinaire HAL, est destinée au dépôt et à la diffusion de documents scientifiques de niveau recherche, publiés ou non, émanant des établissements d'enseignement et de recherche français ou étrangers, des laboratoires publics ou privés.

\section{다)(1) $(5$}

Distributed under a Creative Commons Attribution - NonCommercial| 4.0 International 


\title{
Q-plates micro-arrays for parallel processing of the photon orbital angular momentum
}

\author{
Charles Loussert, Kateryna Kushnir, and Etienne Brasselet ${ }^{\mathrm{a})}$ \\ Laboratoire Ondes et Matière d'Aquitaine, CNRS, Univ. Bordeaux, F 33400 Talence, France
}

\begin{abstract}
We report on the realization of electrically tunable micro-arrays of space-variant optically anisotropic optical vortex generators. Each individual light orbital angular momentum processor consists of a microscopic self-engineered nematic liquid crystal $q$-plate made of a nonsingular topological defect spontaneously formed under electric field. Both structural and optical characterizations of the obtained spin-orbit optical interface are analyzed. An analytical model is derived and results of simulations are compared with experimental data. The application potential in terms of parallel processing of the optical orbital angular momentum is quantitatively discussed.
\end{abstract}

Shaping the phase of an optical field, if possible in realtime, is a common requirement for many applications such as beam shaping and adaptive optics, to name a few. In particular, to obtain spiraling phase profiles remain a topical challenge since Laguerre-Gaussian beams have been identified as beams carrying a well-defined amount of orbital angular momentum per photon. ${ }^{1}$ Indeed, this opened up a wide range of applications ${ }^{2}$ that promoted the development of various techniques to shape the topology of light fields. Among available strategies, those based on the coupling between the spin and orbital angular momentum of light via space-variant birefringent elements ${ }^{3}$ represent an attractive option. Indeed, this allows polarization-controlled handedness of the spiraling wavefront ${ }^{4}$ that is a key property for both classical and quantum optics issues. ${ }^{5}$

Azimuthally, patterned liquid crystals slabs ${ }^{6,7}$ and nanostructured glass slabs ${ }^{8}$ are nowadays commercially available technologies. Such spin-orbit optical elements are sometimes referred to as " $q$-plates" where the half-integer $q$ defines the azimuthal distribution of the optical axis orientation angle $\psi$ of the form $\psi=q \phi$ with $\phi$ the azimuthal angle. Ideally, the birefringent phase retardation $\Delta$ of a $q$-plate is constant and equals $\pi$ for the operating wavelength. In that case, an incident circularly polarized axisymmetric light beam centered on the $q$-plate emerges from it as an orthogonally polarized vortex beam having an azimuthal dependence of the field amplitude of the form $\exp (i \ell \phi)$, where $\ell= \pm 2 q$ is the topological charge of optical phase singularity and \pm sign depends on the handedness of the incident circular polarization state.

Some of the expected future developments of $q$-plates, such as space-division multiplexing of the orbital angular momentum of light, imply the development of $q$-plates arrays. This requires mastering the fabrication of miniaturized $q$-plates, which is not an easy task. In fact, state-of-the-art optical structuring of the anisotropic properties of a $q$-plate is confronted to inherent difficulties associated with the attempt to fabricate an artificial material singularity. In practice, the required azimuthal patterning is typically lost below ten

\footnotetext{
a)Email: etienne.brasselet@u bordeaux.fr
}

micrometers or so, though recent advances in this direction are worth mentioning. ${ }^{9}$ A few years ago, it has been proposed to use natural topological defects of liquid crystal droplets, hence free of machining techniques, to achieve microscopic spin-orbit optical vortex generators. ${ }^{10}$ Since then, several approaches explored the use of liquid crystal topological defects as self-engineered individual or arrays of small scale $q$-plates. Indeed, one can mention natural, ${ }^{11,12}$ electrical, ${ }^{13,14}$ magnetic, ${ }^{15}$ optical, ${ }^{16-20}$ and electro-opti$\mathrm{cal}^{21,22}$ options to realize microscopic azimuthally polarized birefringent structures. However, the realization of efficient parallel processing of the optical orbital angular momentum at small scale remains elusive to date.

Here, we report on the realization of electrically tunable micro-arrays of $q$-plates individually made of electrically induced nonsingular topological defects spontaneously formed in nematic liquid crystal with negative dielectric anisotropy that is, umbilical defects, originally introduced four decades ago. ${ }^{23}$ The experimental demonstration is supported by an analytical model whose simulations are compared with observations.

Umbilical defects are usually obtained by applying a quasistatic electric field at frequency $f$ along the normal to a thin slab (thickness $L=10-100 \mu \mathrm{m}$ ) of nematic liquid crystal sandwiched between two transparent and conductive ITO (indium tin oxide) coated glass slabs treated to ensure perpendicular anchoring of the liquid crystal. At rest the orientational ground state of the director field $\mathbf{n}$ (that is, the local average molecular orientation of the nematic) is illustrated in Fig. 1(a). If the nematic has a negative relative dielectric anisotropy at frequency $f, \epsilon_{a}(f)<0$, umbilical defects randomly appear in the sample above a threshold voltage difference $U=V$ between the two electrodes. Their structure, analytically described by Rapini ${ }^{23}$ and experimentally characterized in three dimensions in Ref. 24, is sketched in Fig. 1(b). They correspond to defects described by a director field of the form $\mathbf{n}=(\sin \vartheta \cos \psi, \sin \vartheta \sin \psi$, $\cos \vartheta)$ in the Cartesian coordinate system $(x, y, z), z$ being the normal to the nematic film, with $\psi(\phi)=s \phi+\psi_{0}$, $s= \pm 1$ and $\psi_{0}$ being a constant, the azimuthal angle of the director in the $(x, y)$ plane and $\vartheta$ the tilt angle of the director 


\section{Uniform electrode design}

(a)

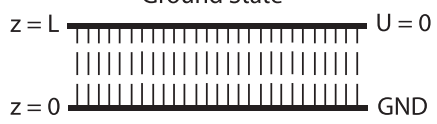

(b)

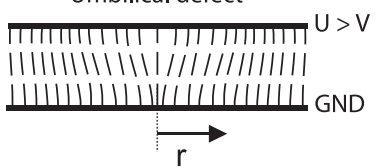

TाTाTाTाTाTाTाTाTाTाTाT $\mathrm{U}>\mathrm{V}$

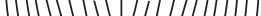
r
Patterned electrode design (c)

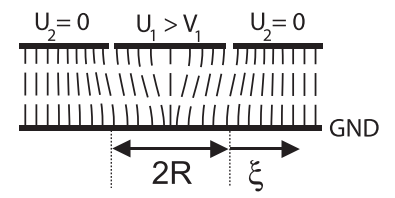

(d) Quench ON

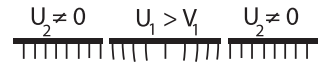
$|1||||||||| 1|||/|||||||||||$ ШШШШШШШШШШШ GND
FIG. 1. (a) Perpendicularly aligned nematic liquid crystal slab with thick ness $L$ at rest. (b) Formation of an umbilical defect at an arbitrary location using uniform electrodes and applying a quasistatic electric field at fre quency $f$ such as the relative dielectric anisotropy is negative, $\epsilon_{a}(f)<0$, for high enough voltage difference $U>V$. (c) and (d) Formation of a localized umbilic in the center of a disk shaped electrode of radius $R$ associated with voltage $U_{1}$ and frequency $f_{1}$, with $\epsilon_{a}\left(f_{1}\right)<0$, surrounded by a region associ ated with $U_{2}, f_{2}$, and $\epsilon_{a}\left(f_{2}\right)>0$. When $U_{2} / 0$, the reorientation tail in region 2 can be quenched, namely, $\xi \rightarrow 0$.

with respect to the $z$ axis. In the framework of the Rapini's model, ${ }^{23}$ the reorientation angle of an isolated umbilic is expressed as $\vartheta(r, z)=\theta(r) \sin (\pi z / L)$, where $r$ is the distance to the defect core, $\theta(r)$ is the tilt amplitude in the mid-plane $z=L / 2$. As shown in a previous work, such defects behave as electrically tunable microscopic $q$-plates with $q= \pm 1 ;^{13}$ however, (i) without spatial control regarding their localization and (ii) without possibility to obtain dense arrays of them because of unavoidable mutual interaction between adjacent defects.

To overcome such limitations, we propose to use patterned electrodes instead of uniform ones. Our idea is shown in Figs. 1(c) and 1(d) that illustrate how a localized umbilical defect can be obtained by using a two-zone electrode pattern associated with two independent quasistatic electric fields $U_{1}$ and $U_{2}$ at frequencies $f_{1}$ and $f_{2}$. Considering the region 1 as a disk of radius $R$ surrounded by the region 2, one can expect the appearance of an umbilic centered on the region 1 , if $\epsilon_{a}\left(f_{1}\right)<0$ when the applied voltage $U_{1}$ is high enough. If $U_{2}=0$, the reorientation profile slightly extends inside the region 2 over a typical distance $\xi$, see Fig. 1(c). On the other hand, if $U_{2} \neq 0$ and $\epsilon_{a}\left(f_{2}\right)>0$, which is possible using a socalled dual frequency nematic material, the reorientation in the region 2 can be electrically quenched, namely, $\xi \rightarrow 0$, thereby preventing the self-engineered microscopic $q$-plate from elastic interaction with its environment.

By extending the Rapini's derivation to the axisymmetric patterned electrode design shown in Figs. 1(c) and 1(d), we obtain the following analytical description for a localized umbilic in the case $\epsilon_{a}\left(f_{1}\right)<0$ and $\epsilon_{a}\left(f_{2}\right)>0$. Namely, the tilt angle amplitude $\theta(r)$ is found to satisfy the nonlinear second order differential equation

$$
\frac{\partial^{2} \theta}{\partial \rho^{2}}+\frac{1}{\rho} \frac{\partial \theta}{\partial \rho}+\left(\frac{1}{\rho_{i}^{2}}-\frac{1}{\rho^{2}}\right) \theta-\frac{1}{\rho_{i}^{2} \Theta_{i}^{2}} \theta^{3}=0,
$$

associated with the boundary conditions $\theta(0)=0$ and $\theta(r \gg R)=0$ and where $\rho=r / R$ is the reduced radial coordinate. In addition, we introduced the square of the reduced distances, $\rho_{i}^{2}$, and reorientation amplitudes, $\Theta_{i}^{2}$, where $i=(1,2)$ refers to the region $i$

$$
\begin{gathered}
\rho_{i}^{2}=\left(\frac{L}{\pi R}\right)^{2}\left[\frac{K_{3}}{K_{2}}\left((-1)^{i+1} \tilde{U}_{i}^{2}-1\right)\right]^{1}, \\
\Theta_{i}^{2}=2 \frac{(-1)^{i+1} \tilde{U}_{i}^{2}-1}{\frac{K_{1}}{K_{3}}+(-1)^{i} \frac{\epsilon_{a}\left(f_{i}\right)}{\epsilon_{\|}\left(f_{i}\right)} \tilde{U}_{i}^{2}},
\end{gathered}
$$

where $\tilde{U}_{i}=U_{i} / V_{i}$ is the reduced voltage with $V_{i}=\pi$ $\left[K_{3} /\left(\epsilon_{0}\left|\epsilon_{a}\left(f_{i}\right)\right|\right)\right]^{1 / 2}, \epsilon_{\|}\left(f_{i}\right)$ is the relative dielectric constant parallel to the director, and $K_{1}, K_{2}$, and $K_{3}$ are the usual splay, twist, and bend elastic constants of the nematic. ${ }^{25}$ We notice that Eq. (2) implies to deal with an umbilic of the twist type, ${ }^{23}$ that is, $\psi_{0}=\pi / 2$, in agreement with the fact that $K_{2}<K_{1}$ for the nematic material used in our experiments.

The simulations are made by using the material parameters of the dual-frequency nematic liquid crystal MLC-2048 (from Merck), namely, $\epsilon_{a}\left(f_{1}\right)=-3.4$ at $f_{1}=100 \mathrm{kHz}$, $\epsilon_{a}\left(f_{2}\right)=3.22$ at $f_{2}=1 \mathrm{kHz}, K_{1}=17.3 \mathrm{pN}, K_{2}=12.7 \mathrm{pN}$, and $K_{3}=30.3 \mathrm{pN}$ (Ref. 26) that give $V_{1} \simeq 3.15 \mathrm{~V}_{\mathrm{rms}}$ and $V_{2} \simeq 3.24 \mathrm{~V}_{\text {rms }}$. In addition, we use cell thickness $L=13 \mu \mathrm{m}$ and electrode radius $R=30 \mu \mathrm{m}$ that correspond to the values of our experimental investigations. The radial profile $\theta$ is shown in Fig. 2(a) for various values of $\tilde{U}_{1}$ when $\tilde{U}_{2}=0$, whereas the effect of the quenching voltage $\tilde{U}_{2}$ on the director reorientation in region 2 at fixed $\tilde{U}_{1}$ is shown in Fig. 2(b). The efficiency of the electrical screening strategy regarding the elastic interaction of a localized umbilical defect with possible nearby defects is inferred from the dependence of the characteristic length $\xi$ introduced in Fig. $1(\mathrm{c})$ on $\tilde{U}_{2}$, see Fig. 2(c), where $\xi$ is defined as $\theta(R+\xi)=0.1 \max _{r}[\theta(r)]$.
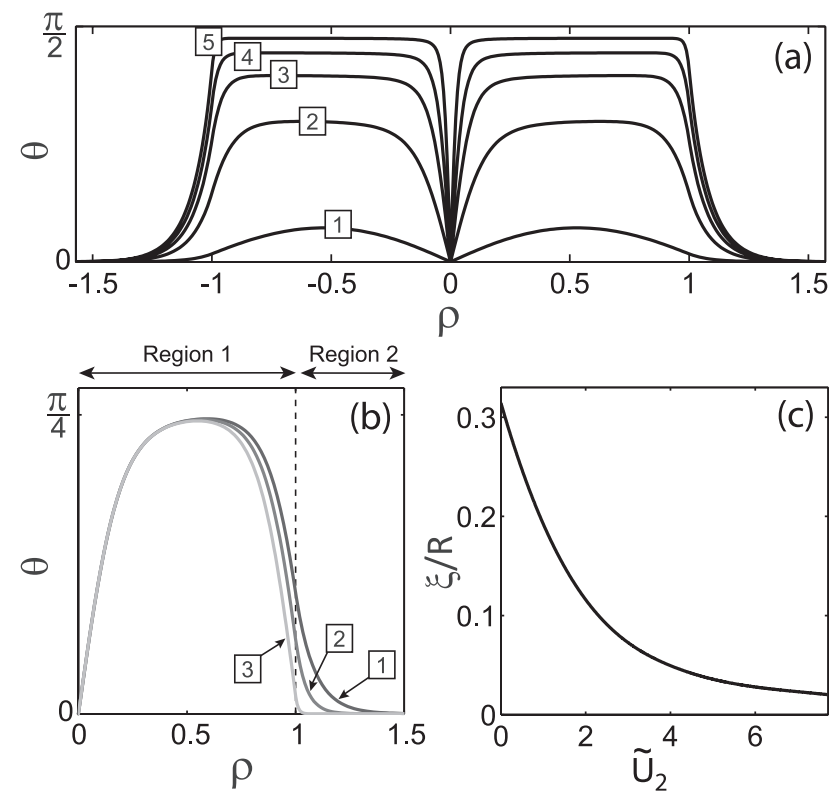

FIG. 2. (a) Simulated reorientation amplitude $\theta$ vs reduced coordinate $\rho \quad r / R$ for $U_{1} 1.0626$ (curve 1), 1.43 (curve 2), 2.06 (curve 3), 3.01 (curve 4), and 5.55 (curve 5) at $U_{2} \quad$ 0. (b) Electrical quenching of the director reorientation in region 2 at fixed $U_{1} \quad 1.27$, for $U_{2} \quad 0$ (curve 1), 1.54 (curve 2), and 7.72 (curve 3). (c) Dependence of the characteristic length $\xi$ [see Fig. 1(c)] on the reduced quenching voltage $U_{2}$. 



FIG. 3. (a) Map of the spin orbit conversion efficiency $\eta$ versus reduced beam waist $w / R$ and applied voltage $U_{1}$ at $U_{2} \quad 0$, where the circle marker indicates the maximal value $\eta_{\max } \quad 0.89$ obtained for $w / R \quad 0.69$ and $U_{1} \quad 1.92$. The dependence of $\eta$ along the dashed lines shown in panel (a) are shown in panels (b) and (c). In panel (b), the index $p$ refers to peak number. In panel (c), the grey area refers to a $10 \%$ deviation from $\eta_{\max }$.

The ability of a defect to shape the wavefront topology of an incident light field is evaluated from the power fraction $\eta$ of the contra-circularly polarized component of the output light field when the defect is illuminated on-axis by a circularly polarized Gaussian beam. ${ }^{13}$ From previous work, see Eqs. (11) and (12) in Ref. 14, one gets

$$
\eta=\frac{4}{w^{2}} \int_{0}^{\infty} \exp \left(-2 r^{2} / w^{2}\right) \sin ^{2}[\Delta(r) / 2] r d r,
$$

where $w$ is the beam waist radius in the plane of the cell, $\Delta(r)=\frac{2 \pi}{\lambda} \int_{0}^{L}\left[n_{e}(r, z)-n_{\perp}\right] d z$ is the total birefringent retardation acquired by light as it passed throughout the film, with $n_{e}=n_{\|} n_{\perp} /\left[n_{\|}^{2} \cos ^{2} \vartheta(r, z)+n_{\perp}^{2} \sin ^{2} \vartheta(r, z)\right]^{1 / 2}$, the effective local refractive index of the extraordinary wave and $n_{\|, \perp}$ the refractive index parallel and perpendicular to the director. The calculated map of $\eta$ in the plane of parameters $\left(w / R, \tilde{U}_{1}\right)$ at $\tilde{U}_{2}=0$ is shown in Fig. 3(a). A global maximum efficiency $\eta_{\max }=0.89$ is found for $w / R=0.69$ and $\tilde{U}_{1}=1.92$. The dependence of $\eta$ versus reduced beam waist $w / R$ at fixed $\tilde{U}_{1}=1.92$ and applied voltage $\tilde{U}_{1}$ at fixed $w / R=0.69$, see dashed lines in Fig. 3(a), are shown in Figs. 3 (c) and 3(b), respectively. The latter plots allow a quantitative assessment of the sensitivity of the optimal spin-orbit behavior, as emphasized by the grey area in Fig. 3(c) that refers to $10 \%$ variation around maximum value $\eta_{\max }$.

Experimental investigation of the proposed electrically tunable micro-arrays of liquid crystals $q$-plates concept is carried out by using a $L=13 \mu \mathrm{m}$-thick nematic film sandwiched between two ITO-coated glass substrates treated with cetyl-trimethyl-ammonium bromide surfactant (from Merck) providing with perpendicular anchoring. Electrode patterning is achieved by direct laser ablation performed before assembling the liquid crystal cell (see for instance Ref. 27). The chosen micro-pattern consists in onedimensional array of $2 R=60 \mu \mathrm{m}$-side square electrodes that defines region 1, as illustrated in Fig. 4(a), where the black line refers to $e=2 \mu \mathrm{m}$ width ablation line, $d=5 \mu \mathrm{m}$, and $h=5 \mu \mathrm{m}$. The electric quenching of the liquid crystal reorientation in region 2 is inferred from the zero transmission when the sample is image between crossed polarizers, either linear [Fig. 4(b)] or circular [Fig. 4(c)]. The topological strength of the localized umbilic is found to be $s=+1$ in all cases. Indeed, the four-arm dark crosses in Fig. 4(b) indicate $|s|=1$ whereas co-rotation of the dark cross when rotating the crossed polarizers implies $s>0$. We also note the presence of a squeezed umbilic with strength $s=-1$ in the narrow channel that connects the square pad to the bus line. On the other hand, the dark spot in the center of each square in Fig. 4(c) is reminiscent of the generation of an optical phase singularity, here with topological charge $\ell= \pm 2$ since $q=s=+1$, see Fig. 4(d) where the phase profile of the generated vortex field determined from spatially resolved polarimetric analysis ${ }^{13}$ is shown.

The optical vortex generation efficiency $\eta$ of a given localized umbilic is measured at $532 \mathrm{~nm}$ wavelength according to the setup depicted in Fig. 5(a) following the procedure detailed in Ref. 13. Typical intensity and phase distribution of the generated optical vortex with topological charge two are shown in Figs. 5(b) and 5(c), respectively. The dependence of $\eta$ as a function of $\tilde{U}_{1}$ at fixed $w / R=1$ is shown in Fig. 5(d), which gives $\eta_{\max } \simeq 0.96$ at $\tilde{U}_{1} \simeq 3.0$ whereas the influence of $w / R$ is shown in Fig. 5(e) $\tilde{U}_{1} \simeq 3.0$. We note that, strictly speaking, the data for $\eta$ above $w / R \simeq 1$ do not correspond to a single defect since the beam thus overlaps significantly with adjacent defects. A trade-off for optimal efficiency while probing a single-defect, therefore, implies to use a typical value $w / R \simeq 1 / 2$, which qualitatively agree with the predicted results, see Fig. 3(c).

The application potential of such arrays in terms of parallel processing of the optical orbital angular momentum practically implies to have independently controlled microscopic $q$-plates. Indeed, since the operating wavelength of a single umbilic can be electrically tuned as shown in Ref. 13, this would allow to achieve optimized efficiency for any set (a)

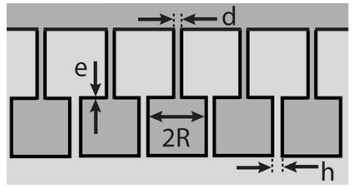

(b)

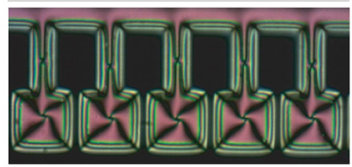

(c)

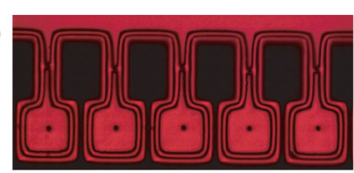

(d)



FIG. 4. (a) Patterned electrode design used in experiments. Umbilical defects observed between crossed linear polarizers (under white light illumi nation) and crossed circular polarizers (under $633 \mathrm{~nm}$ wavelength illumina tion) at $U_{1} \simeq 3$ and $U_{2} \quad 25 \mathrm{~V}_{\mathrm{rms}}$ are shown in panels (b) and (c), respectively. (d) Phase profile of the generated vortex field with topological charge two. Scale bar is $60 \mu \mathrm{m}$. 
(a)



(d)

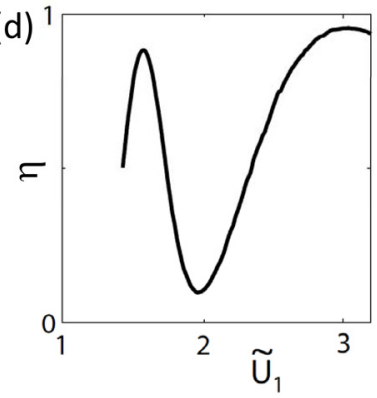

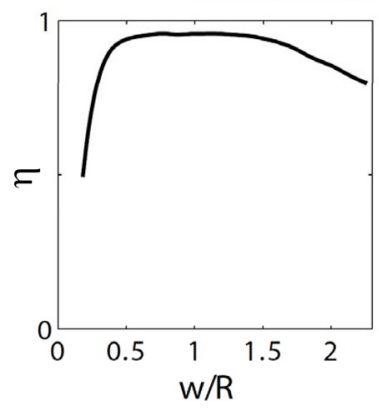

(e)

FIG. 5. (a) Experimental set up for the measurement of the spin orbit conver sion efficiency $\eta$, where $\mathbf{E}_{\sigma}^{\text {in }}{ }_{ \pm 1}$ refer to incident left/right handed circularly polarized incident Gaussian field with beam waist radius $w_{0} \quad 1.3 \mu \mathrm{m}$. (b) and (c) Typical intensity and phase spatial profiles of the output vortex field $\mathbf{E}_{-\sigma}^{\text {out }}$. (d) $\eta$ vs $U_{1}$ at $U_{2} \quad 25 \mathrm{~V}_{\text {rms }}$ and $w / R \quad 1$, which gives $\eta_{\max } \simeq 0.96$ at $U_{1} \simeq 3.0$. (e) $\eta$ vs $w / R$ at $U_{1} \simeq 3.0$.
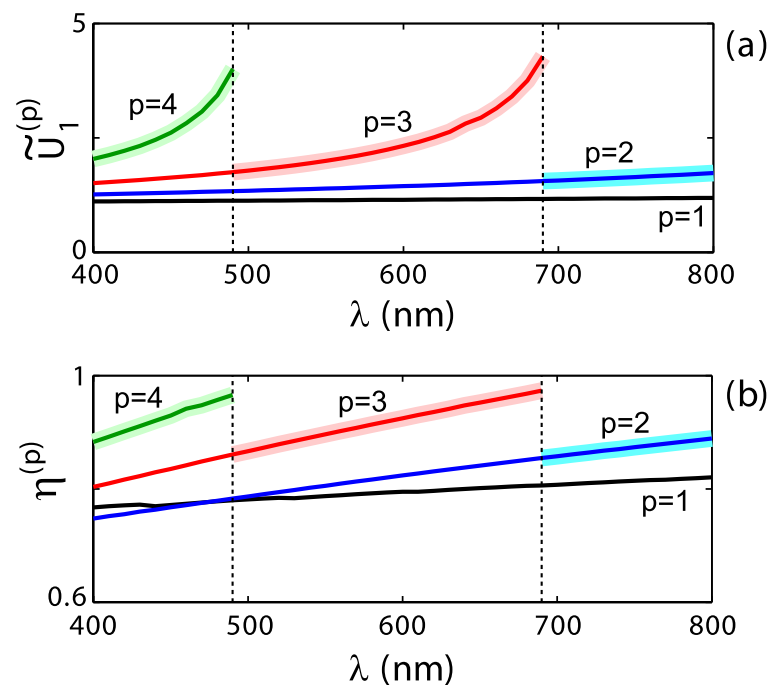

FIG. 6. (a) Calculated discrete set of reduced voltage values $U_{1}^{(p)}$ that corre spond to the $p$ th peak value of the voltage dependent $\eta$, see Fig. 3(c). (b) Corresponding values $\eta^{(p)}$. Thick parts of curves refer to the optimal behav ior over the visible domain. Parameters: $w / R \quad 0.69$ and $U_{2} \quad 0$.

of optical channels with different wavelength over ultrabroadband domain that is not accessible to a single element. Although individual electrical tuning implies an appropriate electrode design, our model allows us to evaluate the spectral capabilities of the proposed micro-arrays. This is done in the visible domain using $w / R=0.69$ and $U_{2}=0$. The discrete set of $\tilde{U}_{1}^{(p)}$ values that correspond to the $p$ th peak value of $\eta$ [see Fig. 3(c)] are shown in Fig. 6(a), whereas the corresponding values $\eta^{(p)}$ are shown in Fig. 6(b). In these figures,

the vertical dashed lines refer to cut-off values for local maxima of $\eta$ and the optimal situation for every wavelength is highlighted by thick parts of curves. An efficiency larger than $85 \%$ is obtained over the visible domain.

To summarize, we have reported on the realization of efficient electrically tunable one-dimensional micro-arrays of $q$-plates made of spontaneously formed liquid crystal topological defects. Extension to two-dimensional arrays can be straightforwardly considered by appropriate electrode patterning. The proposed self-engineered approach allows considering microscopic spin-orbit optical elements while preserving efficient topological shaping ability. This opens up the use of $q$-plates towards the hyperspectral management of the orbital angular momentum of light.

We acknowledge DGA Contract No. 201160083.

${ }^{1}$ L. Allen, M. W. Beijersbergen, R. J. C. Spreeuw, and J. P. Woerdman, Phys. Rev. A 45, 8185 (1992).

${ }^{2}$ D. L. Andrews, Structured Light and Its Applications: An Introduction to Phase Structured Beams and Nanoscale Optical Forces (Academic Press Elsevier, Burlington, 2008).

${ }^{3}$ G. Biener, A. Niv, V. Kleiner, and E. Hasman, Opt. Lett. 27, 1875 (2002).

${ }^{4}$ L. Marrucci, C. Manzo, and D. Paparo, Phys. Rev. Lett. 96, 163905 (2006).

${ }^{5}$ L. Marrucci, E. Karimi, S. Slussarenko, B. Piccirillo, E. Santamato, E. Nagali, and F. Sciarrino, J. Opt. 13, 064001 (2011).

${ }^{6}$ S. Nersisyan, N. Tabiryan, D. M. Steeves, and B. R. Kimball, Opt. Express 17, 11926 (2009).

${ }^{7}$ S. Slussarenko, A. Murauski, T. Du, V. Chigrinov, L. Marrucci, and E. Santamato, Opt. Express 19, 4085 (2011).

${ }^{8}$ M. Beresna, M. Gecevicius, P. G. Kazansky, and T. Gertus, Appl. Phys. Lett. 98, 201101 (2011).

${ }^{9}$ S. R. Nersisyan, N. V. Tabiryan, D. Mawet, and E. Serabyn, Opt. Express 21, 8205 (2013).

${ }^{10}$ E. Brasselet, N. Murazawa, H. Misawa, and S. Juodkazis, Phys. Rev. Lett. 103, 103903 (2009).

${ }^{11}$ C. Loussert, U. Delabre, and E. Brasselet, Phys. Rev. Lett. 111, 037802 (2013).

${ }^{12}$ B. Son, S. Kim, Y. H. Kim, K. Kalantar, H. M. Kim, H. S. Jeong, S. Q. Choi, J. Shin, H. T. Jung, and Y. H. Lee, Opt. Express 22, 4699 (2014).

${ }^{13}$ E. Brasselet and C. Loussert, Opt. Lett. 36, 719 (2011).

${ }^{14}$ E. Brasselet, Phys. Rev. Lett. 108, 087801 (2012).

${ }^{15}$ P. Pieranski, B. Yang, L. J. Burtz, A. Camu, and F. Simonetti, Liq. Cryst. 40, 1593 (2013).

${ }^{16}$ E. Brasselet, Opt. Lett. 34, 3229 (2009).

${ }^{17}$ E. Brasselet, J. Opt. 12, 124005 (2010).

${ }^{18}$ E. Brasselet, Phys. Rev. A 82, 063836 (2010).

${ }^{19}$ M. E. Ketara and E. Brasselet, Opt. Lett. 37, 602 (2012).

${ }^{20} \mathrm{~B}$. Yang and E. Brasselet, J. Opt. 15, 044021 (2013).

${ }^{21}$ R. Barboza, U. Bortolozzo, G. Assanto, E. Vidal Henriquez, M. G. Clerc, and S. Residori, Phys. Rev. Lett. 109, 143901 (2012).

${ }^{22}$ R. Barboza, U. Bortolozzo, G. Assanto, E. Vidal Henriquez, M. G. Clerc, and S. Residori, Phys. Rev. Lett. 111, 093902 (2013).

${ }^{23}$ A. Rapini, J. Phys. 34, 629 (1973).

${ }^{24}$ M. Gu, I. I. Smalyukh, and O. D. Lavrentovich, Appl. Phys. Lett. 88, 061110 (2006).

${ }^{25}$ P. Oswald and P. Pieransky, Nematic and Cholesteric Liquid Crystals: Concepts and Physical Properties Illustrated by Experiments (Taylor \& Francis, CRC, Boca Raton, FL, 2005).

${ }^{26}$ J. S. Hsu, B. J. Liang, and S. H. Chen, Appl. Phys. Lett. 89, 051920 (2006).

${ }^{27}$ A. Risch and R. Hellmann, Phys. Procedia 12, 133 (2011). 